九州大学学術情報リポジトリ

Kyushu University Institutional Repository

\title{
Investigation of three-dimensional current
} distribution at silver diffusion joint of RE123 coated conductors based on magnetic microscopy combined with finite element method

Higashikawa, Kouhe i

Department of Electrical Engineering, Graduate School of Information Science and Electrical Engineering, Kyushu University

Honda, Yoshihiro

Department of Electrical Engineering, Graduate School of Information Science and Electrical Engineering, Kyushu University

Inoue, Masayoshi

Department of Electrical Engineering, Graduate School of Information Science and Electrical Engineering, Kyushu University

Kiss, Takanobu

Department of Electrical Engineering, Graduate School of Information Science and Electrical Engineering, Kyushu University

他

http://hdl. handle. net/2324/25519

出版情報: IEEE Transactions on Applied Superconductivity. 21 (3)，pp.3403-3407，2011-06. The IEEE Council on Superconductivity

バージョン:

権利関係: (C) 2010 IEEE. Personal use of this material is permitted. Permission from IEEE must be obtained for all other users, including reprinting/ republishing this material for advertising or promotional purposes, creating new collective works for resale or redistribution to servers or lists, or reuse of any copyrighted components of this work in other works. 


\title{
Investigation of Three-Dimensional Current Distribution at Silver Diffusion Joint of RE-123 Coated Conductors Based on Magnetic Microscopy Combined with Finite Element Method
}

\author{
Kohei Higashikawa, Yoshihiro Honda, Masayoshi Inoue, Takanobu Kiss, \\ Noriko Chikumoto, Naomichi Sakai, Teruo Izumi, and Hiroshi Okamoto
}

\begin{abstract}
Combining scanning Hall-probe microscopy (SHPM) with finite element method (FEM), we have investigated three-dimensional current distribution at a silver diffusion joint of RE-123 coated conductor (CC). This research aims at the understanding of electromagnetic behaviors in a jointed $\mathrm{CC}$ and at the establishment of an analysis model for it. Two-dimensional distribution of sheet current density in a jointed sample was visualized by a SHPM measurement with a spatial resolution of a few hundred micrometers. Then, it was found that such a distribution included information about current transfer between the jointed pieces. Furthermore, the experimental results were successfully reconstructed by a three-dimensional FEM analysis. We believe that these will be very important findings for the establishment of jointing processes on $\mathrm{CCs}$ and for the designs of practical applications which need such processes.
\end{abstract}

Index Terms - coated conductor, current distribution, finite element method, joint, scanning Hall-probe microscopy

\section{INTRODUCTION}

$\mathrm{T}$ HE second-generation high temperature superconducting (HTS) tapes such as RE-123 (RE: Y, Gd and so on) coated conductors (CCs) have been developed steadily [1]-[3], and electric power applications comprising CCs have been studied strenuously in particular [4]-[7] from their potential of AC losses reduction [8]-[10]

On the other hand, jointing processes on CCs will be indispensable for such applications considering their maintenance and large scale. Of course, an important

Manuscript received 3 August 2010. This work was supported by the "New Energy and Industrial Technology Development Organization (NEDO) as the Project for Development of Materials \& Power Application of Coated Conductors, M-PACC", "JSPS: KAKENHI (20360143)" and "JSPS: KAKENHI (20.1945)".

K. Higashikawa (+81-92-802-3678, kohei@super.ees.kyushu-u.ac.jp), Y. Honda, M. Inoue and T. Kiss are with the Department of Electrical Engineering, Graduate School of Information Science and Electrical Engineering, Kyushu University, 744 Motooka, Nishi-Ku, Fukuoka 819-0395, Japan.

N. Chikumoto, N. Sakai and T. Izumi are with the Superconductivity Research Laboratory, ISTEC, Tokyo 135-0062, Japan.

H. Okamoto is with the Kyushu Electric Power Co., Inc. Fukuoka 815-8520, Japan. requirement for a joint is low contact resistance. For example, silver diffusion method has been proposed as a low-resistance jointing method [11]. A method for low resistance by removing stabilization layer has also been reported [12]. Furthermore, there are some papers discussing contact resistance of CCs jointed by soldering [13], [14].

However, we should also understand further electromagnetic behaviors beyond contact resistance to improve a jointing process itself as well as to predict its influence on an application. Above all, to clarify current transfer between jointed pieces is important because it is an origin of contact resistance and local heat generation. This means that we need to investigate three-dimensional current distribution at a joint.

In this study, we have investigated current distribution in a jointed sample of GdBCO CC. We devised a special configuration for the joint, and then we could estimate current transfer between the jointed pieces from two-dimensional current distribution obtained by scanning Hall-probe microscopy (SHPM). Furthermore, the experimental results were reconstructed very well by a numerical analysis of three-dimensional finite element method (FEM).

\section{METHOD}

\section{A. Sample}

Fig. 1 shows a schematic and a photograph of the sample. The sample was prepared from two pieces of GdBCO CC with different widths: 5-mm-wide piece and 2-mm-wide piece. The two pieces in contact with each other's silver surface were jointed by silver diffusion method. There were two reasons for the different widths. In case of a joint between multifilamentary CCs, physical interference of the filaments should be avoided from the point of view of coupling loss. Considering the processing accuracy, it will be reasonable to utilize a piece with narrower filaments on one side. The sample corresponded to a basic configuration for that. This was one reason. The other reason was to estimate current sharing ratio for each piece at the joint. If we can do that as a function of longitudinal position, we can estimate current transfer between the pieces. The principle will be described below. 

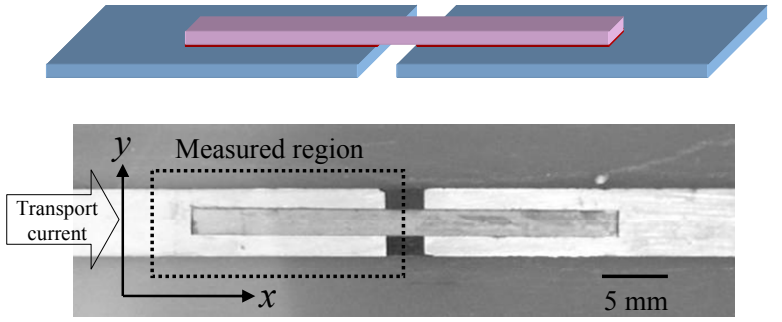

Fig. 1. The schematic and the photograph of the jointed sample of GdBCO CC. Two pieces with different widths were jointed by means of silver diffusion method.

\section{B. Measurement for Sheet Current Density Distribution}

A SHPM system [15], [16] was utilized to visualize current distribution in the sample. Distribution of self-field around the sample induced by a transport current was measured by scanning a Hall-sensor. As a result, in-plane distribution of perpendicular component of the magnetic field, $B_{\mathrm{z}}$, was obtained. If we assume in-plane current distribution in the sample, i.e., sheet current density in $x y$-plane, $J$, the distribution of $\boldsymbol{J}$ can be derived analytically from that of $B_{z}$ by solving an inverse problem of Biot-Savart law. Roth et al. have already reported a method for that, and $x$ and $y$ components of $\boldsymbol{J}, J_{x}$ and $J_{y}$, are expressed in Fourier space as follows [17]:

$$
\begin{aligned}
& \widetilde{J}_{x}\left(k_{x}, k_{y}\right)=-i \frac{2}{\mu_{0}} \frac{k_{y}}{k} e^{k z_{\text {lifi-off }}} \widetilde{B}_{z}\left(k_{x}, k_{y}\right) \\
& \widetilde{J}_{y}\left(k_{x}, k_{y}\right)=i \frac{2}{\mu_{0}} \frac{k_{x}}{k} e^{k z_{\text {lit-off }}} \widetilde{B}_{z}\left(k_{x}, k_{y}\right)
\end{aligned}
$$

where $\widetilde{J}_{x}, \widetilde{J}_{y}$ and $\widetilde{B}_{z}$ are the Fourier transformations of $J_{x}, J_{y}$ and $B_{z}$, respectively. The variables $k_{x}$ and $k_{y}$ are the components of the wavenumber $\boldsymbol{k}$, and $k$ is the absolute value of $\boldsymbol{k}$. The constant $z_{\text {lift-off }}$ is the distance from the current sheet to the measurement point of $B_{z}$. According to the increment of $z_{\text {lift-off, }}$ spatial harmonics of $\widetilde{B}_{z}$ are weakened especially at large $k$. Eqs. (1) and (2) indicate that such harmonics should be amplified by the term of $e^{k z_{\text {liti-off }}}$ to restore the magnetic field just at the current sheet. However, at very large $k$, the harmonics are weakened down to a noise level of the measurement. This means that we should give up the information of such large $k$. We used a Hanning window, $w(k)$, as a low-pass filter:

$$
w(k)=\left\{\begin{array}{cl}
\frac{1+\cos \left(\frac{k}{k_{\text {cut-off }}} \pi\right)}{2} & \text { for } k \leq k_{\text {cut-off }} \\
0 & \text { for } k>k_{\text {cut-off }}
\end{array} .\right.
$$

The parameter $k_{\text {cut-off }}$ is the cut-off wavenumber, and the corresponding cut-off wavelength, $\lambda_{\text {cut-off }}\left(=2 \pi / k_{\text {cut-off }}\right)$, determines the spatial resolution of the obtained distribution of $\boldsymbol{J}$. In this way, in-plane distribution of sheet current density in the sample can be obtained by a SHPM measurement as a superposed distribution of the jointed pieces.

\section{Numerical Analysis}

A commercial FEM software (PHOTO-Series EDDY) was used for an electromagnetic analysis for the sample. Only the 1 - $\mu \mathrm{m}$-thick superconducting layer and the $20-\mu \mathrm{m}$-thick silver layer of the GdBCO CC were considered for the analysis model, and then its nonconductive substrate was not included there. For the superconducting layer, we considered the following electric field versus current density $(E-J)$ characteristics based on the percolation transition model [18], [19]:

$$
E=\left\{\begin{array}{cc}
\frac{\rho_{\mathrm{FF}}}{m+1} J_{0}\left(\frac{J-J_{\mathrm{cm}}}{J_{0}}\right)^{m+1} & \text { for } J \geq J_{\mathrm{cm}} \\
0 & \text { for } J<J_{\mathrm{cm}}
\end{array}\right.
$$

where $\rho_{\mathrm{FF}}=1.0 \times 10^{-7} \Omega \mathrm{m}$ is the resistivity at uniform flux flow, and $J_{\mathrm{cm}}=1.8 \times 10^{10} \mathrm{~A} / \mathrm{m}^{2}, J_{0}=9.1 \times 10^{10} \mathrm{~A} / \mathrm{m}^{2}$ and $m=4.0$ are the parameters describing the shape of the distribution of local critical current density. The software was customized to be able to deal with the $E-J$ equation. Furthermore, we used a time-dependent model for the analysis. The transport current was increased to an intended value by 1 second, and was kept for 3 hours. This was the same condition as the measurement.

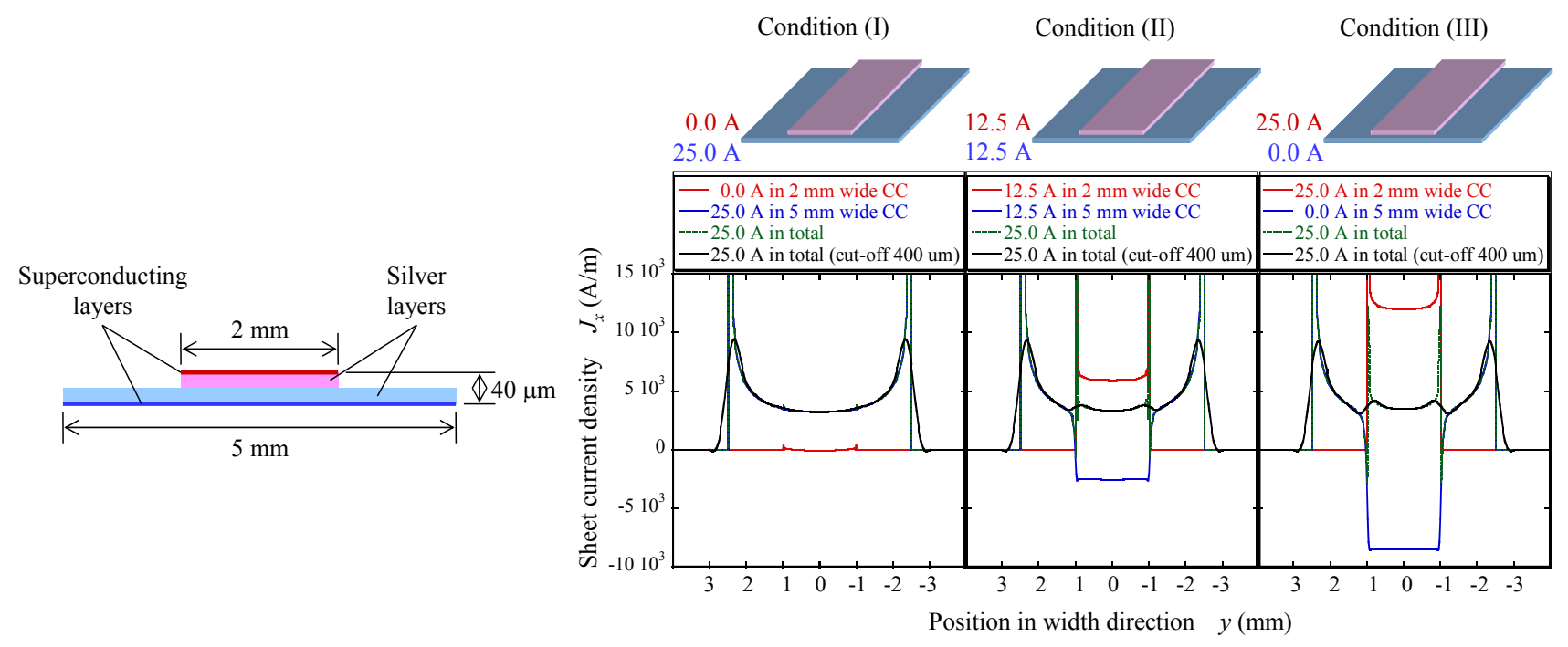

Fig. 2. Theoretical results of the current distribution in the cross-section of the joint of the sample. The results were obtained for different current sharing ratios. The shape of the total distribution changes depending on the current sharing ratio. This means that we can estimate the current value for each piece from a SHPM measurement. 


\section{Estimation of Current Sharing Ratio}

We can only obtain superposed distribution of sheet current density of the jointed pieces by a SHPM measurement. However, according to a theoretical analysis, information of current sharing ratio should be included in the total distribution of sheet current density. Fig. 2 shows the theoretical results. The analysis was carried out for the cross-section at the joint of the sample, and the results were obtained for different current sharing ratios at a total transport current of $25 \mathrm{~A}$. When all the current flows in the 5-mm-wide piece, i.e., in Condition (I), almost no interaction between the two pieces can be seen. On the other hand, when the current becomes larger in the 2-mm-wide piece, shielding current is induced in the 5-mm-wide piece. In Conditions (II) and (III), for example, current flows in negative direction in the 5-mm-wide piece just under the 2-mm-wide piece carrying positive transport current. This is to shield the magnetic field generated by the 2-mm-wide piece. However, the shape of the distribution of the shielding current in the 5-mm-wide piece is slightly different from that of the transport current in the 2-mm-wide piece. For example, the shielding current is induced also outside the region of $-1 \mathrm{~mm} \leq y \leq 1 \mathrm{~mm}$ in the 5 -mm-wide piece while the transport current can flow only within such region in the 2-mm-wide piece. This comes from the distance between the superconducting layers of the two pieces. As a result, the total distribution of sheet current density becomes different shape depending on the current sharing ratio. Even if considering the resolution of a SHPM measurement, i.e., applying (3) with $\lambda_{\text {cut-off }}=400 \mu \mathrm{m}$, such information can remain: local maximum and minimum values around $y= \pm 1 \mathrm{~mm}$. Then, if we plot a difference of the sheet current density between $y=0.8 \mathrm{~mm}$ and $y=1.2 \mathrm{~mm}, \Delta J_{x}$, as a function of the current of 2-mm-wide piece, $I_{2 \mathrm{mmw}}$, we can obtain the following relationship at a total transport current of $25 \mathrm{~A}$ :

$$
I_{2 \mathrm{mmw}}=25 \mathrm{~A} \times \frac{\left(\Delta J_{x}+350 \mathrm{~A} / \mathrm{m}\right)}{1380 \mathrm{~A} / \mathrm{m}} .
$$

By this principle, we can estimate the current sharing ratio at the joint. In other words, we can evaluate current transfer between the two pieces from the SHPM measurement result.

\section{RESUlTS AND DisCUSSION}

\section{A. Results from the SHPM Measurement}

Fig. 3 shows the results from the SHPM measurement. Two-dimensional distribution of magnetic field was measured at a transport current of $25 \mathrm{~A}$, and then the corresponding distribution of sheet current density was obtained with the special resolution of $200 \mu \mathrm{m}: \lambda_{\text {cut-off }}=400 \mu \mathrm{m}$. The $x$ and $y$ components and the absolute value of sheet current density are shown in the figure. It can be seen that current flows largely along the edge of the sample as in the case of a single superconductor. As a result, one may recognize that current transfers from the 5-mm-wide piece to the 2-mm-wide piece intensively on the end of the 5-mm-wide piece: around $x=14$ $\mathrm{mm}$. However, as stated above, this distribution corresponds to the superposed one of the two jointed pieces. Therefore, more discussion will be needed to understand the current transfer.



Fig. 3. Results from the SHPM measurement. Two-dimensional distribution of sheet current density, $\boldsymbol{J}$, was obtained from that of measured magnetic field, $B_{z}$. $J_{\mathrm{x}}$ and $J_{\mathrm{y}}$ are $x$ and $y$ components of $\boldsymbol{J}$, respectively. The measured region is shown in Fig. 1.

\section{B. Current Transfer between the Jointed Pieces}

For the estimation of the current transfer between the jointed pieces, it is necessary to separate the total transport current into those of the two pieces. According to the above-mentioned theoretical analysis, some information about the current sharing ratio should be included in the results of the SHPM measurement. Fig. 4 shows one-dimensional distributions of longitudinal component of sheet current density obtained for several longitudinal positions: $x=3,5,7,9,11 \mathrm{~mm}$. The positions are also indicated in Fig. 3. It can be seen that the shapes of the distributions are different from each other depending on the position: difference between the local maximum and minimum values around $y= \pm 1 \mathrm{~mm}$. As stated above, the current sharing ratio can be estimated by (5). Fig. 5 shows the result. The current in the 5-mm-wide piece, $I_{5 \mathrm{mmw}}$, and that in the 2-mm-wide piece, $I_{2 \mathrm{mmw}}$, are plotted as a function of longitudinal position. Contrary to the appearance of the current distribution shown in Fig. 3, it was found that current transfers from the 5-mm-wide piece to the 2-mm-wide piece by using almost all the length of the joint. Furthermore, $I_{2 \mathrm{mmw}}$ increases linearly with respect to the longitudinal position. This means that current transfers uniformly across the joint length. Therefore, it can be concluded that a decent joint process was performed on this sample. In this way, we could estimate current distribution in a jointed sample beyond two-dimensional distribution. 


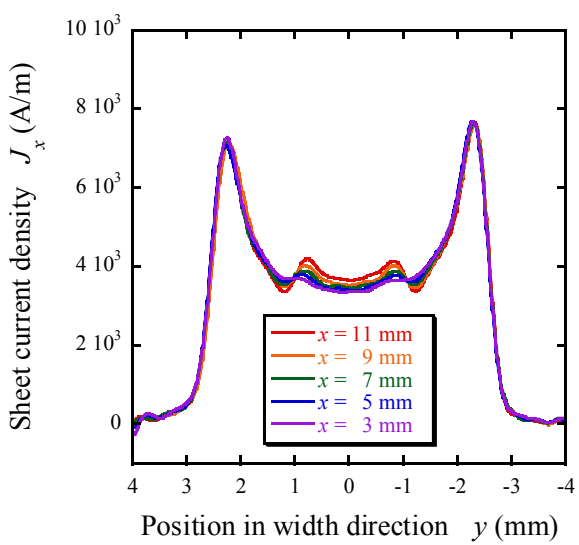

Fig. 4. One-dimensional distributions of sheet current density at several longitudinal positions. These distributions are extracted from the two-dimensional one shown in Fig. 3.



Fig. 5. Current sharing at the joint estimated from the SHPM measurement as a function of longitudinal position. This means current transfer from 5-mm-wide piece to 2-mm-wide piece.

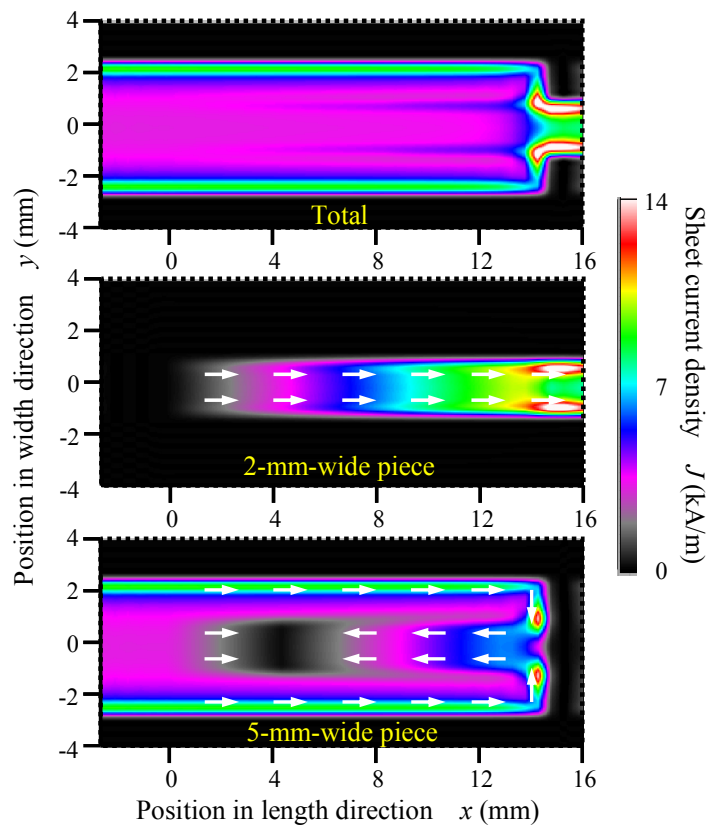

Fig. 6. Results from the 3D FEM analysis. The spatial resolution was matched with that of the SHPM measurement by applying (3). The total distribution of sheet current density agrees well with that of the SHPM measurement shown in Fig. 3.

\section{Results from 3D FEM Analysis}

To confirm the validity of the above-mentioned discussion and to understand further electromagnetic behaviors in the sample, we also carried out a three-dimensional FEM analysis. Fig. 6 shows the results. Current distributions in the 5-mm-wide piece and the 2-mm-wide piece are shown in the figure. The superposed distribution of both pieces is also shown for the comparison with the experimental result. It can be seen that current in the 2-mm-wide piece gradually increases by using all the length of the joint as discussed in the experimental results. Furthermore, it should be noted that current flows in negative direction in the 5-mm-wide piece just under the 2-mm-wide piece. This is the shielding current against the magnetic field induced by the 2 -mm-wide piece. As a result, almost all the information of the current in the 2-mm-wide piece disappears in the total distribution of the two pieces, and then the sample pretends to be a single superconductor. On the other hand, irregularity around $y= \pm 1 \mathrm{~mm}$ still remains in the total distribution. This comes from slight difference between the current distribution in the 2-mm-wide piece and the shielding current in the 5-mm-wide piece due to the distance between the superconducting layers of the two pieces. Including these features, the total distribution agrees very well with the experimental one shown in Fig. 3. Therefore, it can be concluded that we could confirm the validity of the discussion in this paper and that we could establish an analysis model at a joint of CC.

\section{CONCLUSION}

We have investigated three-dimensional current distribution at a silver diffusion joint of GdBCO CC. Two-dimensional distribution of sheet current density in the sample was visualized by a SHPM measurement. Then, it was found that such a distribution included information about current transfer between the jointed pieces, and current transferred from one piece to the other by using almost all the length of the joint. Furthermore, the experimental results were successfully reconstructed by a three-dimensional FEM analysis. We believe that these will be very important findings for the establishment of jointing processes on CCs and for the designs of practical applications which need such processes.

\section{REFERENCES}

[1] V. Selvamanickam, Y. Chen, X. Xiong, Y. Xie, X. Zhang, A. Rar, M. Martchevskii, R. Schmidt, K. Lenseth, and J. Herrin, "Progress in second-generation HTS wire development and manufacturing," Physica $C$, vol. 468, pp. 1504-1509, 2008.

[2] M. W. Rupich, X.P. Li, C. Thieme, S. Sathyamurthy, S. Fleshler, D. Tucker, E. Thompson, J. Schreiber, J. Lynch, D. Buczek, K. DeMoranville, J. Inch, P. Cedrone, and J. Slack, "Advances in second generation high temperature superconducting wire manufacturing and R\&D at American Superconductor Corporation," Supercond. Sci. Technol., vol. 23, pp. 014015 1-9, 2010.

[3] Y. Shiohara, N. Fujiwara, H. Hayashi, S. Nagaya, T. Izumi, and M. Yoshizumi, "Japanese efforts on coated conductor processing and its power applications: new 5 year project for materials and power applications of coated conductors (M-PACC)," Physica C, vol. 469, pp. 863-867, 2009.

[4] K. Shikimachi, N. Hirano, S. Nagaya, H. Kawashima, K. Higashikawa, and T. Nakamura, "System Coordination of 2 GJ Class YBCO SMES for Power System Control," IEEE Trans. Appl. Supercond., vol. 19, no. 2, pp. 2012-2018, 2009 
[5] M. Iwakuma, H. Hayashi, H. Okamoto, A. Tomioka, M. Konno, T. Saito, Y. Iijima, Y. Suzuki, S. Yoshida, Y. Yamada, T. Izumi, and Y. Shiohara, "Development of REBCO superconducting power transformers in Japan," Physica C, vol. 469, pp. 1726-1732, 2009.

[6] S. Mukoyama, M. Yagi, T. Masuda, N. Amemiya, A. Ishiyama, N. Kashima, S. Nagaya e, Y. Aoki f, M. Yoshizumi, Y. Yamada, T. Izumi, and Y. Shiohara, "Development of (RE)BCO cables for HTS power transmission lines," Physica C, vol. 469, pp. 1688-1692, 2009.

[7] H. Yumura, M. Watanabe, M. Ohya, Y. Ashibe, H. Ito, T. Masuda, and K. Sato, "30 m YBCO Cable for the Albany HTS Cable Project," Journal of Physics: Conference Series, vol. 97, pp.012076_1-6, 2008.

[8] C. B. Cobb, P. N. Barnes, T. J. Haugan, J. Tolliver, E. Lee, M. Sumption, E. Collings, and C. E. Oberly, "Hysteretic loss reduction in striated YBCO," Physica C, vol. 382, pp. 52-56, 2002.

[9] N. Amemiya, S. Kasai, K. Yoda, Z. Jiang, G. A. Levin, P. N. Barnes, and C. E. Oberly, "AC loss reduction of YBCO coated conductors by multifilamentary structure," Supercond. Sci. Technol., vol. 17, pp. 1464-1471, 2004.

[10] K. Suzuki, J. Matsuda, M. Yoshizumi, T. Izumi, Y. Shiohara, M. Iwakuma, A. Ibi, S. Miyata, and Y. Yamada, "Development of a laser scribing process of coated conductors for the reduction of AC losses," Supercond. Sci. Technol., vol. 20, pp. 822-826, 2007.

[11] J.Y. Kato, N. Sakai, S. Tajima, S. Miyata, M. Konishi, Y. Yamada, N. Chikumoto, K. Nakao, T. Izumi, and Y. Shiohara, "Diffusion joint of YBCO coated conductors using stabilizing silver layers,", Physica $C$, vol. 445-448, pp. 686-688, 2006.

[12] K. S. Chang, H. Kim, D. K. Park, T. K. Ko, M. C. Ahn, D.-H. Ha, J.-B. Song, S. J. Lee, H. M. Kim, and H. Lee, "Joint Characteristics of YBCO
Coated Conductor by Removing a Metallic Stabilizer," IEEE Trans. Appl. Supercond., vol. 18, no. 2, pp. 1220-1223, 2008.

[13] M. Polak, P. N. Barnes, and G. A. Levin, "YBCO/Ag boundary resistivity in YBCO tapes with metallic substrates," Supercond. Sci. Technol., vol. 19, pp. 817-820, 2006.

[14] D. K. Park, M. C. Ahn, H. M. Kim, H. G. Lee, K. S. Chang, S. J. Lee, S. E. Yang, and T. K. Ko, "Analysis of a Joint Method Between Superconducting YBCO Coated Conductors," IEEE Trans. Appl. Supercond., vol. 17, no. 2, pp. 3266-3269, 2007.

[15] M. Inoue, K. Abiru, Y. Honda, T. Kiss, Y. Iijima, K. Kakimoto, T. Saitoh, K. Nakao, and Y. Shiohara, "Observation of Current Distribution in High-Tc Superconducting Tape Using Scanning Hall-Probe Microscope," IEEE Trans. Appl. Supercond,, vol. 19, no. 3, pp. 2847-2850, 2009.

[16] K. Higashikawa, Y. Honda, M. Inoue, M. Iwakuma, T. Kiss, K. Nakao, Y. Yamada, and T. Izumi, "Spatially-resolved measurement on time-dependent electromagnetic behavior in alternating current carrying coated conductor," Physica $C$, in press.

[17] B. J. Roth, N. G. Sepulveda, and J. P. Wikswo Jr, "Using a magnetometer to image a two-dimensional current distribution," J. Appl. Phys., vol. 65, pp. 361-372, 1989.

[18] K. Yamafuji, and T. Kiss, "Current-voltage characteristics near the glass-liquid transition in high- $T_{\mathrm{c}}$ superconductors," Physica $C$, vol. 290, pp. 9-22, 1997.

[19] T. Kiss, T. Nakamura, N. Mishiro, K. Hasegawa, M. Inoue, M. Takeo, F. Irie, and K. Yamafuji, "Transport characteristics in high $T_{\mathrm{c}}$ superconductors," Proc. MT15, no. 2, pp. 1052-1055, 1998. 